Acta Crystallographica Section E

Structure Reports

Online

ISSN 1600-5368

Charlotte K. Leech, ${ }^{\text {a Alastair J. }}$ Florence, ${ }^{b_{*}}$ Kenneth Shankland, $^{\mathrm{a}}$ Norman Shankland ${ }^{b}$ and Andrea Johnston $^{\text {b }}$

${ }^{a}$ ISIS Facility, Rutherford Appleton Laboratory, Chilton, Didcot, Oxon OX11 0QX, England, and ${ }^{\mathbf{b}}$ Solid-State Research Group, Strathclyde Institute of Pharmacy and Biomedical Sciences, University of Strathclyde, 27 Taylor Street,

Glasgow G4 ONR, Scotland

Correspondence e-mail:

alastair.florence@strath.ac.uk

\section{Key indicators}

Single-crystal $\mathrm{X}$-ray study

$T=150 \mathrm{~K}$

Mean $\sigma(\mathrm{C}-\mathrm{C})=0.003 \AA$

$R$ factor $=0.041$

$w R$ factor $=0.101$

Data-to-parameter ratio $=12.6$

For details of how these key indicators were automatically derived from the article, see http://journals.iucr.org/e.

\title{
10,11-Dihydrocarbamazepine (form III)
}

The title compound (systematic name: 10,11-dihydro-5Hdibenz $[b, f]$ azepine-5-carboxamide), $\mathrm{C}_{15} \mathrm{H}_{14} \mathrm{~N}_{2} \mathrm{O}$, is shown to crystallize as a triclinic polymorph with $Z^{\prime}=2$. N-H $\cdots \mathrm{O}$ and $\mathrm{N}-\mathrm{H} \cdots \pi$ interactions combine to create a catemeric motif. The robustness of this motif is reflected in the fact that it is also observed in the previously published monoclinic and orthorhombic forms of the compound.

\section{Comment}

Dihydrocarbamazepine (DHC), (I), is a recognized impurity in carbamazepine, a dibenzazepine drug used to control seizures (Cyr et al., 1987). DHC is known to crystallize in three polymorphic forms: monoclinic form I $\left[P 2_{1} / c ; a=5.505\right.$ (1) $\AA$,

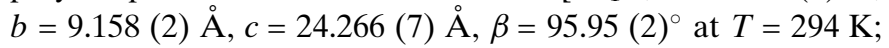
Bandoli et al., 1992], orthorhombic form II [Pbca; $a=$ 9.0592 (4) $\AA, b=10.3156$ (5) $\AA, c=25.0534$ (12) $\AA$ at $T=$ $120 \mathrm{~K}$; Harrison et al., 2006] and triclinic form III (present work). It also forms a 1:1 solvate with acetic acid (Johnston et al., 2006). The work reported here forms part of a wider investigation that couples automated parallel crystallization (Florence, Johnston, Fernandes et al., 2006) with crystal structure prediction methodology to investigate the basic science underlying the solid-state diversity of carbamazepine and its analogues (Florence, Johnston, Price et al., 2006).

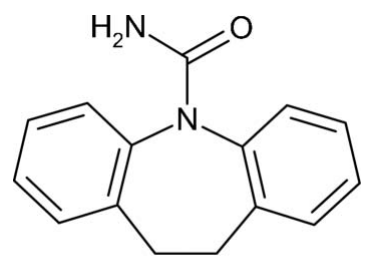

(I)

There are two independent molecules in DHC form III (Fig. 1). The intermolecular interactions combine to create the catemeric motif shown in Fig. 2, with the geometric para-

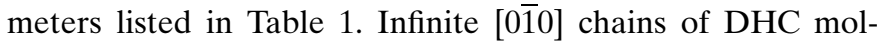
ecules are linked by hydrogen bonds $\mathrm{N} 4-\mathrm{H} 4 B \cdots \mathrm{O} 1$ and $\mathrm{N} 2-\mathrm{H} 2 B \cdots \mathrm{O} 2^{\mathrm{i}}$ [symmetry code: (i) $x, y-1, z$ ], supplemented by $\mathrm{N}-\mathrm{H} \cdots \pi$ interactions, $\mathrm{N} 2-\mathrm{H} 2 A \cdots C g 4$ and $\mathrm{N} 4-$ $\mathrm{H} 4 A \cdots C g 2^{\text {ii }}$ [symmetry code: (ii) $x, y+1, z$ ], where $C g 4$ is the centroid of ring $R 4(\mathrm{C} 29-\mathrm{C} 34)$ and $C g 2$ is the centroid of ring $R 2$ (C9-C14). The robustness of this motif is reflected in the fact that it is observed in DHC form II [Fig. 2 of Harrison et al. (2006)], DHC form I [Fig. 3 of Bandoli et al. (1992)] and in a predicted carbamazepine crystal structure that is isostructural with DHC form II [Fig. 2 of Florence, Leech et al. (2006)]. This
Received 25 October 2006 Accepted 9 December 2006 

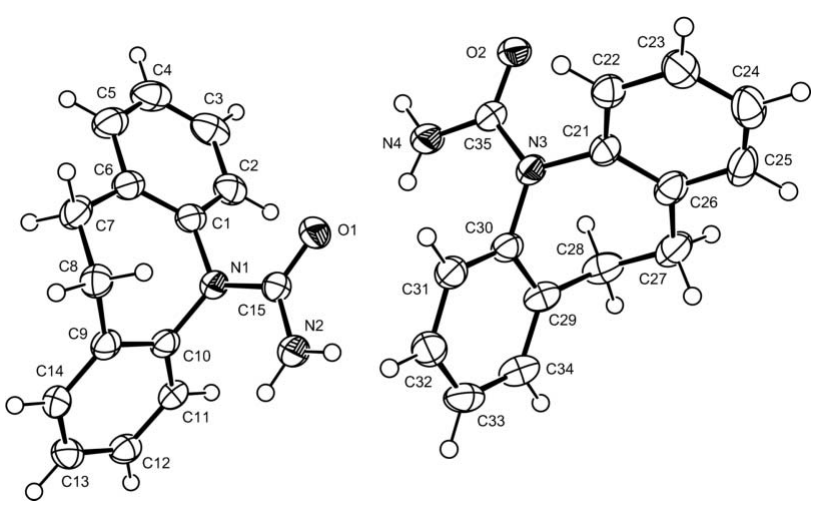

Figure 1

The asymmetric unit of DHC form III with $50 \%$ probability displacement ellipsoids.

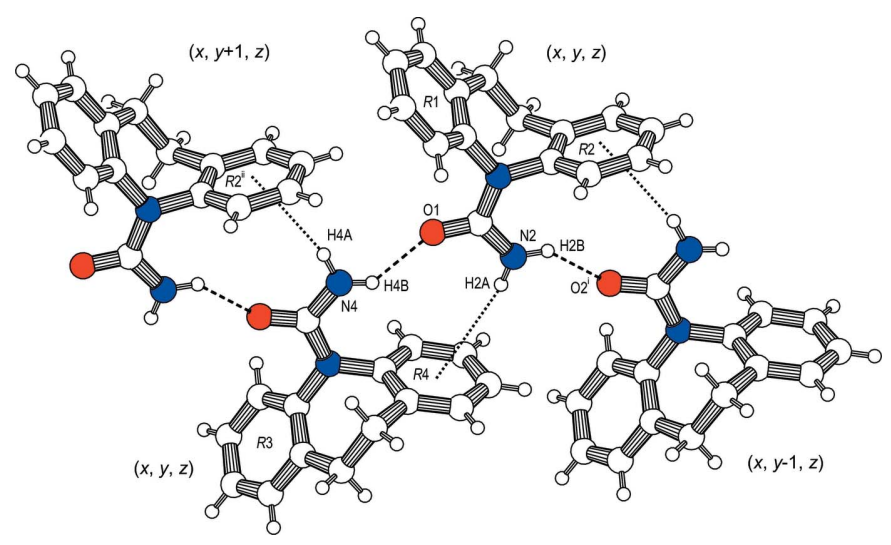

Figure 2

The DHC catemer in form III. Dashed and dotted lines indicate $\mathrm{N}-$ $\mathrm{H} \cdots \mathrm{O}$ and $\mathrm{N}-\mathrm{H} \cdots \pi$ interactions, respectively.

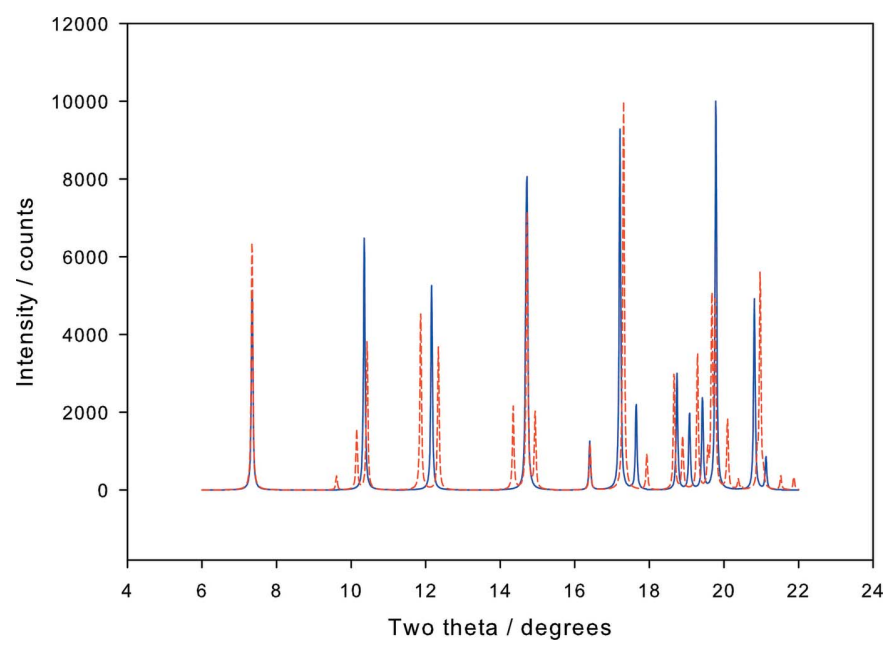

Figure 3

Calculated powder diffraction patterns $(\lambda=1.54 \AA$ ) for DHC form I (blue solid line) and form III (red dashed line).

motif is also observed in the crystal structure of cyheptamide (Leech et al., 2007), an analogue of DHC.
The structures of DHC forms I and III are closely related, but certainly distinct, and there is no evidence of missing symmetry in the form III structure [using the ADDSYM algorithm in PLATON (Spek, 2003)]. Powder patterns calculated from single-crystal structures offer an effective means of distinguishing polymorphs (Karami et al., 2006) and, in this case, the calculated patterns are quite different, reflecting the small but significant differences in both the lattice parameters and the atomic positions (Fig. 3).

\section{Experimental}

DHC was recrystallized from methanol solution by slow evaporation at room temperature to yield single crystals of form I (blocks), form II (hexagonal plates) and form III (needles).

\section{Crystal data}

$\begin{array}{ll}\mathrm{C}_{15} \mathrm{H}_{14} \mathrm{~N}_{2} \mathrm{O} & V=1199.6(8) \AA^{3} \\ M_{r}=238.28 & Z=4 \\ \text { Triclinic, } P \overline{1} & D_{x}=1.319 \mathrm{Mg} \mathrm{m}^{-3} \\ a=5.4233(12) \AA & \mathrm{Cu} \mathrm{K \alpha \text {radiation }} \\ b=9.200(5) \AA & \mu=0.67 \mathrm{~mm}^{-1} \\ c=24.189(6) \AA & T=150(2) \mathrm{K} \\ \alpha=87.59(3)^{\circ} & \text { Needle, colourless } \\ \beta=84.23(2)^{\circ} & 0.22 \times 0.07 \times 0.07 \mathrm{~mm} \\ \gamma=88.93(3)^{\circ} & \end{array}$

\section{Data collection}

Oxford Diffraction Gemini diffractometer

$\omega$ and $\varphi$ scans

Absorption correction: multi-scan

(CrysAlis RED; Oxford

Diffraction, 2006)

$T_{\min }=0.867, T_{\max }=0.955$

\section{Refinement}

Refinement on $F^{2}$

$R\left[F^{2}>2 \sigma\left(F^{2}\right)\right]=0.041$

$w R\left(F^{2}\right)=0.101$

$S=0.84$

4297 reflections

341 parameters 4297 independent reflections 2327 reflections with $I>2 \sigma(I)$

$R_{\text {int }}=0.044$

$\theta_{\max }=67.5^{\circ}$

$$
\begin{aligned}
& \mathrm{H} \text { atoms treated by a mixture of } \\
& \text { independent and constrained } \\
& \text { refinement } \\
& w=1 /\left[\sigma^{2}\left(F_{\mathrm{o}}^{2}\right)+(0.0472 P)^{2}\right] \\
& \text { where } P=\left(F_{\mathrm{o}}{ }^{2}+2 F_{\mathrm{c}}{ }^{2}\right) / 3 \\
& (\Delta / \sigma)_{\max }<0.001 \\
& \Delta \rho_{\max }=0.22 \AA^{-3} \\
& \Delta \rho_{\min }=-0.17 \mathrm{e}^{-3}
\end{aligned}
$$

Table 1

Hydrogen-bond geometry $\left(\AA \mathrm{A}^{\circ}\right)$.

\begin{tabular}{lllll}
\hline$D-\mathrm{H} \cdots A$ & $D-\mathrm{H}$ & $\mathrm{H} \cdots A$ & $D \cdots A$ & $D-\mathrm{H} \cdots A$ \\
\hline $\mathrm{N} 2-\mathrm{H} 2 B \cdots \mathrm{O} 2^{\mathrm{i}}$ & $0.92(2)$ & $2.02(3)$ & $2.800(3)$ & $142.7(19)$ \\
$\mathrm{N} 4-\mathrm{H} 4 B \cdots \mathrm{O} 1$ & $0.86(2)$ & $2.11(3)$ & $2.801(3)$ & $137.4(19)$ \\
$\mathrm{N} 2-\mathrm{H} 2 A \cdots C g 4$ & $0.89(3)$ & $3.01(3)$ & $3.862(3)$ & $162(2)$ \\
$\mathrm{N} 4-\mathrm{H} 4 A \cdots C g 2^{\mathrm{ii}}$ & $0.90(3)$ & $2.89(3)$ & $3.765(3)$ & $166(2)$ \\
\hline
\end{tabular}

Symmetry codes: (i) $x, y-1, z$; (ii) $x, y+1, z$.

The amide $\mathrm{H}$ atoms were located in difference maps and their coordinates and $U_{\text {iso }}$ parameters refined freely. All other $\mathrm{H}$ atoms were constrained to geometrically sensible positions in a riding model, with $\mathrm{C}-\mathrm{H}=0.95-0.99 \AA$ and with $U_{\text {iso }}(\mathrm{H})=1.2 U_{\text {eq }}(\mathrm{C})$.

Data collection: CrysAlis CCD (Oxford Diffraction, 2006); cell refinement: CrysAlis RED (Oxford Diffraction, 2006); data reduc- 
tion: CrysAlis RED; program(s) used to solve structure: SHELXS97 (Sheldrick, 1997); program(s) used to refine structure: SHELXL97 (Sheldrick, 1997); molecular graphics: PLATON (Spek, 2003); software used to prepare material for publication: SHELXL97.

We thank the Basic Technology programme of the UK Research Councils for funding under the project Control and Prediction of the Organic Solid State (http://www.cposs.org.uk).

\section{References}

Bandoli, G., Nicolini, M., Onagaro, A., Volpe, G. \& Rubello, A. (1992). J. Chem. Crystallogr. 22, 177-183.

Cyr, T. D., Matsui, F., Sears, R. W., Curran, N. M. \& Lovering, E. G. (1987). J. Assoc. Off. Anal. Chem. 70, 836-840.
Florence, A. J., Johnston, A., Fernandes, P., Shankland, N. \& Shankland, K. (2006). J. Appl. Cryst. 39, 922-924.

Florence, A. J., Johnston, A., Price, S. L., Nowell, H., Kennedy, A. R. \& Shankland, N. (2006). J. Pharm. Sci. 95, 1918-1930.

Florence, A. J., Leech, C. K., Shankland, N., Shankland, K. \& Johnston, A. (2006). CrystEngComm, 8, 746-747.

Harrison, W. T. A., Yathirajan, H. S. \& Anilkumar, H. G. (2006). Acta Cryst. C62, o240-o242.

Johnston, A., Florence, A. J., Fernandes, P., Shankland, N. \& Kennedy, A. R. (2006). Acta Cryst. E62, o5361-05362.

Karami, S., Li, Y., Hughes, D. S., Hursthouse, M. B., Russell, A. E., Threlfall, T. L., Claybourn, M. \& Roberts, R. (2006). Acta Cryst. B62, 689-691.

Leech, C. K., Florence, A. J., Shankland, K., Shankland, N. \& Johnston, A. (2007). Acta Cryst. E63, o205-o206.

Oxford Diffraction (2006). CrysAlis CCD and CrysAlis RED. Versions 1.171.29.2. Oxford Diffraction Ltd, Abingdon, Oxfordshire, England.

Sheldrick, G. M. (1997). SHELXS97 and SHELXL97. University of Göttingen, Germany.

Spek, A. L. (2003). J. Appl. Cryst. 36, 7-13. 


\section{supporting information}

Acta Cryst. (2007). E63, o675-o677 [https://doi.org/10.1107/S1600536806053335]

\section{0,11-Dihydrocarbamazepine (form III)}

\section{Charlotte K. Leech, Alastair J. Florence, Kenneth Shankland, Norman Shankland and Andrea Johnston}

10,11-Dihydrocarbamazepine

\section{Crystal data}

$\mathrm{C}_{15} \mathrm{H}_{14} \mathrm{~N}_{2} \mathrm{O}$

$M_{r}=238.28$

Triclinic, $P \overline{1}$

Hall symbol: -P 1

$a=5.4233$ (12) $\AA$

$b=9.200(5) \AA$

$c=24.189(6) \AA$

$\alpha=87.59(3)^{\circ}$

$\beta=84.23(2)^{\circ}$

$\gamma=88.93(3)^{\circ}$

$V=1199.6(8) \AA^{3}$

\section{Data collection}

Oxford Diffraction Gemini diffractometer

Radiation source: Enhance $(\mathrm{Cu}) \mathrm{X}$-ray Source Graphite monochromator

Detector resolution: 15.9745 pixels $\mathrm{mm}^{-1}$

$\omega$ and $\varphi$ scans

Absorption correction: multi-scan

(CrysAlis RED; Oxford Diffraction, 2006)

$T_{\min }=0.867, T_{\max }=0.955$

\section{Refinement}

Refinement on $F^{2}$

Least-squares matrix: full

$R\left[F^{2}>2 \sigma\left(F^{2}\right)\right]=0.041$

$w R\left(F^{2}\right)=0.101$

$S=0.84$

4297 reflections

341 parameters

0 restraints

Primary atom site location: structure-invariant direct methods
$Z=4$

$F(000)=504$

$D_{\mathrm{x}}=1.319 \mathrm{Mg} \mathrm{m}^{-3}$

$\mathrm{Cu} K \alpha$ radiation, $\lambda=1.54184 \AA$

Cell parameters from 2529 reflections

$\theta=3.7-72.8^{\circ}$

$\mu=0.67 \mathrm{~mm}^{-1}$

$T=150 \mathrm{~K}$

Needle, colourless

$0.22 \times 0.07 \times 0.07 \mathrm{~mm}$

12410 measured reflections

4297 independent reflections

2327 reflections with $I>2 \sigma(I)$

$R_{\text {int }}=0.044$

$\theta_{\max }=67.5^{\circ}, \theta_{\min }=3.7^{\circ}$

$h=-5 \rightarrow 6$

$k=-11 \rightarrow 10$

$l=-28 \rightarrow 28$

Secondary atom site location: difference Fourier map

Hydrogen site location: inferred from neighbouring sites

$\mathrm{H}$ atoms treated by a mixture of independent and constrained refinement

$w=1 /\left[\sigma^{2}\left(F_{\mathrm{o}}^{2}\right)+(0.0472 P)^{2}\right]$

where $P=\left(F_{\mathrm{o}}^{2}+2 F_{\mathrm{c}}^{2}\right) / 3$

$(\Delta / \sigma)_{\max }<0.001$

$\Delta \rho_{\max }=0.22 \mathrm{e} \AA^{-3}$

$\Delta \rho_{\min }=-0.17$ e $\AA^{-3}$ 


\section{Special details}

Geometry. All e.s.d.'s (except the e.s.d. in the dihedral angle between two 1.s. planes) are estimated using the full covariance matrix. The cell e.s.d.'s are taken into account individually in the estimation of e.s.d.'s in distances, angles and torsion angles; correlations between e.s.d.'s in cell parameters are only used when they are defined by crystal symmetry. An approximate (isotropic) treatment of cell e.s.d.'s is used for estimating e.s.d.'s involving 1.s. planes.

Refinement. Refinement of $F^{2}$ against ALL reflections. The weighted $R$-factor $w R$ and goodness of fit $S$ are based on $F^{2}$, conventional $R$-factors $R$ are based on $F$, with $F$ set to zero for negative $F^{2}$. The threshold expression of $F^{2}>\sigma\left(F^{2}\right)$ is used only for calculating $R$-factors (gt) etc. and is not relevant to the choice of reflections for refinement. $R$-factors based on $F^{2}$ are statistically about twice as large as those based on $F$, and $R$ - factors based on ALL data will be even larger.

Fractional atomic coordinates and isotropic or equivalent isotropic displacement parameters $\left(\AA^{2}\right)$

\begin{tabular}{|c|c|c|c|c|}
\hline & $x$ & $y$ & $z$ & $U_{\text {iso }} * / U_{\text {eq }}$ \\
\hline O1 & -0.1609 & $0.31475(16)$ & $0.28334(6)$ & $0.0399(4)$ \\
\hline N1 & $-0.3307(4)$ & 0.11654 (19) & $0.33152(7)$ & $0.0324(4)$ \\
\hline N2 & $-0.0524(4)$ & $0.0916(2)$ & $0.25291(8)$ & $0.0426(5)$ \\
\hline $\mathrm{H} 2 \mathrm{~A}$ & $0.060(6)$ & $0.131(3)$ & $0.2277(12)$ & $0.069(9)^{*}$ \\
\hline $\mathrm{H} 2 \mathrm{~B}$ & $-0.042(4)$ & -0.008 & $0.2558(9)$ & $0.034(7)^{*}$ \\
\hline $\mathrm{C} 1$ & $-0.4478(4)$ & $0.2066(2)$ & $0.37405(9)$ & $0.0340(5)$ \\
\hline $\mathrm{C} 2$ & $-0.6367(5)$ & $0.3015(2)$ & $0.35965(10)$ & $0.0395(6)$ \\
\hline $\mathrm{H} 2$ & -0.6800 & 0.3065 & 0.3225 & $0.047 *$ \\
\hline $\mathrm{C} 3$ & $-0.7613(5)$ & 0.3880 & $0.39872(12)$ & $0.0473(7)$ \\
\hline H3 & -0.8903 & 0.4522 & 0.3887 & $0.057^{*}$ \\
\hline $\mathrm{C} 4$ & $-0.6964(5)$ & 0.3805 & $0.45266(11)$ & $0.0530(8)$ \\
\hline $\mathrm{H} 4$ & -0.7793 & 0.4407 & 0.4798 & $0.064 *$ \\
\hline $\mathrm{C} 5$ & $-0.5110(5)$ & 0.2856 & $0.46687(10)$ & $0.0477(7)$ \\
\hline H5 & -0.4709 & 0.2804 & 0.5042 & $0.057^{*}$ \\
\hline C6 & $-0.3802(5)$ & $0.1967(2)$ & $0.42832(9)$ & $0.0364(6)$ \\
\hline $\mathrm{C} 7$ & $-0.1833(5)$ & 0.0965 & $0.44960(9)$ & $0.0419(6)$ \\
\hline H7A & -0.2666 & 0.0288 & 0.4781 & $0.050 *$ \\
\hline H7B & -0.0712 & 0.1566 & 0.4687 & $0.050^{*}$ \\
\hline $\mathrm{C} 8$ & $-0.0225(4)$ & 0.0054 & $0.40825(9)$ & $0.0368(5)$ \\
\hline H8A & 0.0679 & 0.0707 & 0.3799 & $0.044^{*}$ \\
\hline H8B & 0.1015 & -0.0509 & 0.4280 & $0.044^{*}$ \\
\hline $\mathrm{C} 9$ & $-0.1762(4)$ & $-0.0973(2)$ & $0.38008(9)$ & $0.0326(5)$ \\
\hline $\mathrm{C} 10$ & $-0.3336(4)$ & $-0.0384(2)$ & $0.34217(9)$ & $0.0314(5)$ \\
\hline $\mathrm{C} 11$ & $-0.4906(4)$ & $-0.1256(2)$ & $0.31713(9)$ & $0.0327(5)$ \\
\hline H11 & -0.5975 & -0.0840 & 0.2918 & $0.039 *$ \\
\hline $\mathrm{C} 12$ & $-0.4914(4)$ & $-0.2740(2)$ & $0.32916(9)$ & $0.0363(6)$ \\
\hline H12 & -0.5995 & -0.3344 & 0.3121 & $0.044^{*}$ \\
\hline $\mathrm{C} 13$ & $-0.3358(5)$ & $-0.3342(3)$ & $0.36575(9)$ & $0.0390(6)$ \\
\hline H13 & -0.3369 & -0.4362 & 0.3738 & $0.047 *$ \\
\hline $\mathrm{C} 14$ & $-0.1768(4)$ & $-0.2468(2)$ & $0.39110(9)$ & $0.0353(5)$ \\
\hline H14 & -0.0685 & -0.2894 & 0.4160 & $0.042 *$ \\
\hline C15 & -0.1761 (4) & $0.1812(2)$ & $0.28836(9)$ & $0.0314(5)$ \\
\hline $\mathrm{O} 2$ & 0.1329 & $0.81843(16)$ & $0.22084(6)$ & $0.0360(4)$ \\
\hline N3 & $0.3008(4)$ & $0.63384(19)$ & $0.16859(7)$ & $0.0317(4)$ \\
\hline N4 & $0.0134(4)$ & $0.5889(2)$ & $0.24465(9)$ & $0.0426(5)$ \\
\hline
\end{tabular}




$\begin{array}{lllll}\text { H4B } & 0.015(4) & 0.496(3) & 0.2416(9) & 0.036(7)^{*} \\ \text { H4A } & -0.077(5) & 0.626(3) & 0.2740(11) & 0.047(7)^{*} \\ \text { C21 } & 0.4437(4) & 0.7339(2) & 0.13104(9) & 0.0334(5) \\ \text { C22 } & 0.6176(4) & 0.8181(2) & 0.15319(9) & 0.0340(5) \\ \text { H22 } & 0.6339 & 0.8105 & 0.1919 & 0.041^{*} \\ \text { C23 } & 0.7667(5) & 0.9128(2) & 0.11940(10) & 0.0424(6) \\ \text { H23 } & 0.8865 & 0.9691 & 0.1346 & 0.051^{*} \\ \text { C24 } & 0.7391(5) & 0.9241(3) & 0.06339(11) & 0.0462(7) \\ \text { H24 } & 0.8374 & 0.9906 & 0.0399 & 0.055^{*} \\ \text { C25 } & 0.5707(5) & 0.8400(3) & 0.04142(10) & 0.0451(7) \\ \text { H25 } & 0.5588 & 0.8477 & 0.0025 & 0.054^{*} \\ \text { C26 } & 0.4141(5) & 0.7425(3) & 0.07420(9) & 0.0373(6) \\ \text { C27 } & 0.2361(5) & 0.6556(3) & 0.04489(9) & 0.0462(7) \\ \text { H27A } & 0.3361 & 0.5938 & 0.0183 & 0.055^{*} \\ \text { H27B } & 0.1409 & 0.7252 & 0.0227 & 0.055^{*} \\ \text { C28 } & 0.0496(5) & 0.5573(3) & 0.07958(10) & 0.0446(6) \\ \text { H28A } & -0.0582 & 0.6171 & 0.1054 & 0.053^{*} \\ \text { H28B } & -0.0568 & 0.5110 & 0.0546 & 0.053^{*} \\ \text { C29 } & 0.1772(4) & 0.4415(3) & 0.11223(9) & 0.0379(6) \\ \text { C30 } & 0.3084(4) & 0.4827(2) & 0.15580(9) & 0.0323(5) \\ \text { C31 } & 0.4467(4) & 0.3825(2) & 0.18449(9) & 0.0347(5) \\ \text { H31 } & 0.5378 & 0.4129 & 0.2134 & 0.042^{*} \\ \text { C32 } & 0.4515(5) & 0.2380(3) & 0.17082(10) & 0.0410(6) \\ \text { H32 } & 0.5462 & 0.1688 & 0.1903 & 0.049^{*} \\ \text { C33 } & 0.3174(5) & 0.1941(3) & 0.12854(10) & 0.0431(6) \\ \text { H33 } & 0.3190 & 0.0947 & 0.1194 & 0.052^{*} \\ \text { C34 } & 0.1818(5) & 0.2949(3) & 0.09973(10) & 0.0422(6) \\ \text { H34 } & 0.0903 & 0.2639 & 0.0710 & 0.051^{*} \\ \text { C35 } & 0.1453(4) & 0.6875(2) & 0.21248(9) & 0.0313(5) \\ & & & & \end{array}$

Atomic displacement parameters $\left(\AA^{2}\right)$

\begin{tabular}{lllllll}
\hline & $U^{11}$ & $U^{22}$ & $U^{33}$ & $U^{12}$ & $U^{13}$ & $U^{23}$ \\
\hline O1 & $0.0472(11)$ & $0.0317(9)$ & $0.0390(9)$ & $-0.0038(7)$ & $0.0038(8)$ & $0.0007(7)$ \\
N1 & $0.0380(11)$ & $0.0314(10)$ & $0.0269(9)$ & $-0.0025(8)$ & $0.0013(8)$ & $-0.0021(7)$ \\
N2 & $0.0540(15)$ & $0.0362(13)$ & $0.0346(11)$ & $-0.0022(10)$ & $0.0115(10)$ & $-0.0024(9)$ \\
C1 & $0.0359(13)$ & $0.0307(12)$ & $0.0342(12)$ & $-0.0077(10)$ & $0.0047(10)$ & $-0.0034(9)$ \\
C2 & $0.0373(14)$ & $0.0347(13)$ & $0.0457(14)$ & $-0.0063(11)$ & $0.0001(11)$ & $-0.0013(10)$ \\
C3 & $0.0377(14)$ & $0.0351(14)$ & $0.0665(18)$ & $-0.0028(11)$ & $0.0090(13)$ & $-0.0044(12)$ \\
C4 & $0.0562(18)$ & $0.0423(15)$ & $0.0559(17)$ & $-0.0066(13)$ & $0.0231(14)$ & $-0.0146(12)$ \\
C5 & $0.0583(18)$ & $0.0451(15)$ & $0.0381(14)$ & $-0.0129(13)$ & $0.0084(12)$ & $-0.0099(11)$ \\
C6 & $0.0397(14)$ & $0.0346(13)$ & $0.0339(12)$ & $-0.0102(11)$ & $0.0057(10)$ & $-0.0055(10)$ \\
C7 & $0.0509(16)$ & $0.0455(15)$ & $0.0305(12)$ & $-0.0125(12)$ & $-0.0062(11)$ & $-0.0037(10)$ \\
C8 & $0.0368(13)$ & $0.0387(13)$ & $0.0357(13)$ & $-0.0056(11)$ & $-0.0070(10)$ & $-0.0010(10)$ \\
C9 & $0.0334(13)$ & $0.0361(13)$ & $0.0270(12)$ & $-0.0037(10)$ & $0.0042(10)$ & $-0.0030(9)$ \\
C10 & $0.0334(12)$ & $0.0340(12)$ & $0.0258(11)$ & $-0.0016(10)$ & $0.0039(9)$ & $-0.0046(9)$ \\
C11 & $0.0326(13)$ & $0.0384(13)$ & $0.0268(11)$ & $-0.0004(10)$ & $-0.0002(10)$ & $-0.0052(9)$ \\
C12 & $0.0366(14)$ & $0.0372(14)$ & $0.0349(12)$ & $-0.0079(11)$ & $0.0013(11)$ & $-0.0065(10)$
\end{tabular}




$\begin{array}{lllllll}\text { C13 } & 0.0441(15) & 0.0312(13) & 0.0392(13) & -0.0055(11) & 0.0080(11) & -0.0003(10) \\ \text { C14 } & 0.0404(14) & 0.0362(14) & 0.0282(12) & -0.0003(11) & 0.0008(10) & 0.0016(9) \\ \text { C15 } & 0.0337(13) & 0.0316(13) & 0.0294(12) & 0.0006(10) & -0.0060(10) & -0.0016(9) \\ \text { O2 } & 0.0403(10) & 0.0321(9) & 0.0348(9) & -0.0014(7) & 0.0021(7) & -0.0070(6) \\ \text { N3 } & 0.0353(11) & 0.0344(11) & 0.0248(9) & -0.0027(8) & 0.0012(8) & -0.0037(7) \\ \text { N4 } & 0.0537(14) & 0.0351(13) & 0.0365(12) & -0.0049(10) & 0.0111(10) & -0.0069(9) \\ \text { C21 } & 0.0358(13) & 0.0339(13) & 0.0287(11) & 0.0033(10) & 0.0054(10) & -0.0026(9) \\ \text { C22 } & 0.0330(13) & 0.0352(13) & 0.0328(12) & 0.0053(10) & 0.0015(10) & -0.0026(9) \\ \text { C23 } & 0.0386(14) & 0.0380(14) & 0.0479(15) & 0.0009(11) & 0.0074(12) & -0.0004(11) \\ \text { C24 } & 0.0449(16) & 0.0420(15) & 0.0471(15) & 0.0056(12) & 0.0125(12) & 0.0073(11) \\ \text { C25 } & 0.0547(17) & 0.0483(16) & 0.0281(12) & 0.0176(13) & 0.0101(12) & 0.0049(10) \\ \text { C26 } & 0.0399(14) & 0.0416(14) & 0.0297(12) & 0.0117(11) & -0.0008(10) & -0.0040(10) \\ \text { C27 } & 0.0565(17) & 0.0532(16) & 0.0294(12) & 0.0151(13) & -0.0074(12) & -0.0078(11) \\ \text { C28 } & 0.0433(15) & 0.0501(16) & 0.0428(14) & 0.0063(12) & -0.0110(12) & -0.0168(11) \\ \text { C29 } & 0.0372(14) & 0.0413(14) & 0.0352(12) & 0.0035(11) & 0.0006(10) & -0.0118(10) \\ \text { C30 } & 0.0341(13) & 0.0332(13) & 0.0289(11) & -0.0028(10) & 0.0034(10) & -0.0065(9) \\ \text { C31 } & 0.0368(13) & 0.0366(13) & 0.0306(12) & -0.0033(10) & -0.0015(10) & -0.0034(10) \\ \text { C32 } & 0.0417(15) & 0.0384(14) & 0.0407(14) & 0.0055(11) & 0.0056(11) & -0.0014(10) \\ \text { C33 } & 0.0439(15) & 0.0369(14) & 0.0476(15) & 0.0017(12) & 0.0048(12) & -0.0148(11) \\ \text { C34 } & 0.0385(14) & 0.0479(15) & 0.0411(14) & 0.0023(12) & -0.0023(11) & -0.0173(11) \\ \text { C35 } & 0.0309(12) & 0.0353(13) & 0.0277(11) & -0.0010(10) & -0.0018(9) & -0.0052(9) \\ & & & & & & \end{array}$

Geometric parameters $\left(\AA,{ }^{\circ}\right)$

\begin{tabular}{llll}
\hline $\mathrm{O} 1-\mathrm{C} 15$ & $1.233(3)$ & $\mathrm{O} 2-\mathrm{C} 35$ & $1.229(3)$ \\
$\mathrm{N} 1-\mathrm{C} 15$ & $1.392(3)$ & $\mathrm{N} 3-\mathrm{C} 35$ & $1.390(3)$ \\
$\mathrm{N} 1-\mathrm{C} 10$ & $1.438(3)$ & $\mathrm{N} 3-\mathrm{C} 30$ & $1.436(3)$ \\
$\mathrm{N} 1-\mathrm{C} 1$ & $1.439(3)$ & $\mathrm{N} 3-\mathrm{C} 21$ & $1.444(3)$ \\
$\mathrm{N} 2-\mathrm{C} 15$ & $1.337(3)$ & $\mathrm{N} 4-\mathrm{C} 35$ & $1.339(3)$ \\
$\mathrm{N} 2-\mathrm{H} 2 \mathrm{~A}$ & $0.89(3)$ & $\mathrm{N} 4-\mathrm{H} 4 \mathrm{~B}$ & $0.86(2)$ \\
$\mathrm{N} 2-\mathrm{H} 2 \mathrm{~B}$ & $0.92(2)$ & $\mathrm{N} 4-\mathrm{H} 4 \mathrm{~A}$ & $0.90(3)$ \\
$\mathrm{C} 1-\mathrm{C} 2$ & $1.394(3)$ & $\mathrm{C} 21-\mathrm{C} 22$ & $1.393(3)$ \\
$\mathrm{C} 1-\mathrm{C} 6$ & $1.397(3)$ & $\mathrm{C} 21-\mathrm{C} 26$ & $1.399(3)$ \\
$\mathrm{C} 2-\mathrm{C} 3$ & $1.378(3)$ & $\mathrm{C} 22-\mathrm{C} 23$ & $1.383(3)$ \\
$\mathrm{C} 2-\mathrm{H} 2$ & 0.9500 & $\mathrm{C} 22-\mathrm{H} 22$ & 0.9500 \\
$\mathrm{C} 3-\mathrm{C} 4$ & $1.384(4)$ & $\mathrm{C} 23-\mathrm{C} 24$ & $1.377(3)$ \\
$\mathrm{C} 3-\mathrm{H} 3$ & 0.9500 & $\mathrm{C} 23-\mathrm{H} 23$ & 0.9500 \\
$\mathrm{C} 4-\mathrm{C} 5$ & $1.380(4)$ & $\mathrm{C} 24-\mathrm{C} 25$ & $1.369(4)$ \\
$\mathrm{C} 4-\mathrm{H} 4$ & 0.9500 & $\mathrm{C} 24-\mathrm{H} 24$ & 0.9500 \\
$\mathrm{C} 5-\mathrm{C} 6$ & $1.397(3)$ & $\mathrm{C} 25-\mathrm{C} 26$ & $1.410(3)$ \\
$\mathrm{C} 5-\mathrm{H} 5$ & 0.9500 & $\mathrm{C} 25-\mathrm{H} 25$ & 0.9500 \\
$\mathrm{C} 6-\mathrm{C} 7$ & $1.513(3)$ & $\mathrm{C} 26-\mathrm{C} 27$ & $1.512(3)$ \\
$\mathrm{C} 7-\mathrm{C} 8$ & $1.527(3)$ & $\mathrm{C} 27-\mathrm{C} 28$ & $1.531(3)$ \\
$\mathrm{C} 7-\mathrm{H} 7 \mathrm{~A}$ & 0.9900 & $\mathrm{C} 27-\mathrm{H} 27 \mathrm{~A}$ & 0.9900 \\
$\mathrm{C} 7-\mathrm{H} 7 \mathrm{~B}$ & 0.9900 & $\mathrm{C} 27-\mathrm{H} 27 \mathrm{~B}$ & 0.9900 \\
$\mathrm{C} 8-\mathrm{C} 9$ & $1.500(3)$ & $\mathrm{C} 28-\mathrm{C} 29$ & $1.500(3)$ \\
$\mathrm{C} 8-\mathrm{H} 8 \mathrm{~A}$ & 0.9900 & $\mathrm{C} 28-\mathrm{H} 28 \mathrm{~A}$ & 0.9900 \\
$\mathrm{C} 8-\mathrm{H} 8 \mathrm{~B}$ & 0.9900 & $\mathrm{C} 28-\mathrm{H} 28 \mathrm{~B}$ & 0.9900 \\
& & &
\end{tabular}




\begin{tabular}{|c|c|c|c|}
\hline $\mathrm{C} 9-\mathrm{C} 14$ & $1.390(3)$ & $\mathrm{C} 29-\mathrm{C} 34$ & $1.394(3)$ \\
\hline $\mathrm{C} 9-\mathrm{C} 10$ & $1.400(3)$ & $\mathrm{C} 29-\mathrm{C} 30$ & $1.398(3)$ \\
\hline $\mathrm{C} 10-\mathrm{C} 11$ & $1.380(3)$ & $\mathrm{C} 30-\mathrm{C} 31$ & $1.385(3)$ \\
\hline $\mathrm{C} 11-\mathrm{C} 12$ & $1.384(3)$ & $\mathrm{C} 31-\mathrm{C} 32$ & $1.382(3)$ \\
\hline $\mathrm{C} 11-\mathrm{H} 11$ & 0.9500 & $\mathrm{C} 31-\mathrm{H} 31$ & 0.9500 \\
\hline $\mathrm{C} 12-\mathrm{C} 13$ & $1.376(3)$ & $\mathrm{C} 32-\mathrm{C} 33$ & $1.391(3)$ \\
\hline $\mathrm{C} 12-\mathrm{H} 12$ & 0.9500 & $\mathrm{C} 32-\mathrm{H} 32$ & 0.9500 \\
\hline $\mathrm{C} 13-\mathrm{C} 14$ & $1.393(3)$ & $\mathrm{C} 33-\mathrm{C} 34$ & $1.382(4)$ \\
\hline $\mathrm{C} 13-\mathrm{H} 13$ & 0.9500 & $\mathrm{C} 33-\mathrm{H} 33$ & 0.9500 \\
\hline $\mathrm{C} 14-\mathrm{H} 14$ & 0.9500 & C34-H34 & 0.9500 \\
\hline $\mathrm{C} 15-\mathrm{N} 1-\mathrm{C} 10$ & $121.98(18)$ & $\mathrm{C} 35-\mathrm{N} 3-\mathrm{C} 30$ & $122.25(18)$ \\
\hline $\mathrm{C} 15-\mathrm{N} 1-\mathrm{C} 1$ & $118.82(18)$ & $\mathrm{C} 35-\mathrm{N} 3-\mathrm{C} 21$ & $119.51(18)$ \\
\hline $\mathrm{C} 10-\mathrm{N} 1-\mathrm{C} 1$ & $117.67(17)$ & $\mathrm{C} 30-\mathrm{N} 3-\mathrm{C} 21$ & $117.98(17)$ \\
\hline $\mathrm{C} 15-\mathrm{N} 2-\mathrm{H} 2 \mathrm{~A}$ & $117.4(18)$ & $\mathrm{C} 35-\mathrm{N} 4-\mathrm{H} 4 \mathrm{~B}$ & $127.7(15)$ \\
\hline $\mathrm{C} 15-\mathrm{N} 2-\mathrm{H} 2 \mathrm{~B}$ & $127.7(14)$ & $\mathrm{C} 35-\mathrm{N} 4-\mathrm{H} 4 \mathrm{~A}$ & $114.1(15)$ \\
\hline $\mathrm{H} 2 \mathrm{~A}-\mathrm{N} 2-\mathrm{H} 2 \mathrm{~B}$ & $113(2)$ & $\mathrm{H} 4 \mathrm{~B}-\mathrm{N} 4-\mathrm{H} 4 \mathrm{~A}$ & $118(2)$ \\
\hline $\mathrm{C} 2-\mathrm{C} 1-\mathrm{C} 6$ & $121.0(2)$ & $\mathrm{C} 22-\mathrm{C} 21-\mathrm{C} 26$ & $121.2(2)$ \\
\hline $\mathrm{C} 2-\mathrm{C} 1-\mathrm{N} 1$ & $117.7(2)$ & $\mathrm{C} 22-\mathrm{C} 21-\mathrm{N} 3$ & $117.6(2)$ \\
\hline $\mathrm{C} 6-\mathrm{C} 1-\mathrm{N} 1$ & $121.3(2)$ & $\mathrm{C} 26-\mathrm{C} 21-\mathrm{N} 3$ & $121.2(2)$ \\
\hline $\mathrm{C} 3-\mathrm{C} 2-\mathrm{C} 1$ & $120.7(3)$ & $\mathrm{C} 23-\mathrm{C} 22-\mathrm{C} 21$ & $120.8(2)$ \\
\hline $\mathrm{C} 3-\mathrm{C} 2-\mathrm{H} 2$ & 119.6 & $\mathrm{C} 23-\mathrm{C} 22-\mathrm{H} 22$ & 119.6 \\
\hline $\mathrm{C} 1-\mathrm{C} 2-\mathrm{H} 2$ & 119.6 & $\mathrm{C} 21-\mathrm{C} 22-\mathrm{H} 22$ & 119.6 \\
\hline $\mathrm{C} 2-\mathrm{C} 3-\mathrm{C} 4$ & $119.3(3)$ & $\mathrm{C} 24-\mathrm{C} 23-\mathrm{C} 22$ & $119.0(3)$ \\
\hline $\mathrm{C} 2-\mathrm{C} 3-\mathrm{H} 3$ & 120.4 & $\mathrm{C} 24-\mathrm{C} 23-\mathrm{H} 23$ & 120.5 \\
\hline $\mathrm{C} 4-\mathrm{C} 3-\mathrm{H} 3$ & 120.4 & $\mathrm{C} 22-\mathrm{C} 23-\mathrm{H} 23$ & 120.5 \\
\hline $\mathrm{C} 5-\mathrm{C} 4-\mathrm{C} 3$ & $119.9(2)$ & $\mathrm{C} 25-\mathrm{C} 24-\mathrm{C} 23$ & $120.3(2)$ \\
\hline $\mathrm{C} 5-\mathrm{C} 4-\mathrm{H} 4$ & 120.1 & $\mathrm{C} 25-\mathrm{C} 24-\mathrm{H} 24$ & 119.9 \\
\hline $\mathrm{C} 3-\mathrm{C} 4-\mathrm{H} 4$ & 120.1 & $\mathrm{C} 23-\mathrm{C} 24-\mathrm{H} 24$ & 119.9 \\
\hline $\mathrm{C} 4-\mathrm{C} 5-\mathrm{C} 6$ & $122.4(3)$ & $\mathrm{C} 24-\mathrm{C} 25-\mathrm{C} 26$ & $122.7(2)$ \\
\hline $\mathrm{C} 4-\mathrm{C} 5-\mathrm{H} 5$ & 118.8 & $\mathrm{C} 24-\mathrm{C} 25-\mathrm{H} 25$ & 118.6 \\
\hline $\mathrm{C} 6-\mathrm{C} 5-\mathrm{H} 5$ & 118.8 & $\mathrm{C} 26-\mathrm{C} 25-\mathrm{H} 25$ & 118.6 \\
\hline $\mathrm{C} 5-\mathrm{C} 6-\mathrm{C} 1$ & $116.7(2)$ & $\mathrm{C} 21-\mathrm{C} 26-\mathrm{C} 25$ & $116.0(2)$ \\
\hline $\mathrm{C} 5-\mathrm{C} 6-\mathrm{C} 7$ & $116.9(2)$ & $\mathrm{C} 21-\mathrm{C} 26-\mathrm{C} 27$ & $126.4(2)$ \\
\hline $\mathrm{C} 1-\mathrm{C} 6-\mathrm{C} 7$ & $126.3(2)$ & $\mathrm{C} 25-\mathrm{C} 26-\mathrm{C} 27$ & $117.6(2)$ \\
\hline $\mathrm{C} 6-\mathrm{C} 7-\mathrm{C} 8$ & $118.9(2)$ & $\mathrm{C} 26-\mathrm{C} 27-\mathrm{C} 28$ & $119.1(2)$ \\
\hline $\mathrm{C} 6-\mathrm{C} 7-\mathrm{H} 7 \mathrm{~A}$ & 107.6 & $\mathrm{C} 26-\mathrm{C} 27-\mathrm{H} 27 \mathrm{~A}$ & 107.6 \\
\hline $\mathrm{C} 8-\mathrm{C} 7-\mathrm{H} 7 \mathrm{~A}$ & 107.6 & $\mathrm{C} 28-\mathrm{C} 27-\mathrm{H} 27 \mathrm{~A}$ & 107.6 \\
\hline $\mathrm{C} 6-\mathrm{C} 7-\mathrm{H} 7 \mathrm{~B}$ & 107.6 & $\mathrm{C} 26-\mathrm{C} 27-\mathrm{H} 27 \mathrm{~B}$ & 107.6 \\
\hline $\mathrm{C} 8-\mathrm{C} 7-\mathrm{H} 7 \mathrm{~B}$ & 107.6 & $\mathrm{C} 28-\mathrm{C} 27-\mathrm{H} 27 \mathrm{~B}$ & 107.6 \\
\hline $\mathrm{H} 7 \mathrm{~A}-\mathrm{C} 7-\mathrm{H} 7 \mathrm{~B}$ & 107.0 & $\mathrm{H} 27 \mathrm{~A}-\mathrm{C} 27-\mathrm{H} 27 \mathrm{~B}$ & 107.0 \\
\hline $\mathrm{C} 9-\mathrm{C} 8-\mathrm{C} 7$ & $111.3(2)$ & $\mathrm{C} 29-\mathrm{C} 28-\mathrm{C} 27$ & $111.6(2)$ \\
\hline $\mathrm{C} 9-\mathrm{C} 8-\mathrm{H} 8 \mathrm{~A}$ & 109.4 & $\mathrm{C} 29-\mathrm{C} 28-\mathrm{H} 28 \mathrm{~A}$ & 109.3 \\
\hline $\mathrm{C} 7-\mathrm{C} 8-\mathrm{H} 8 \mathrm{~A}$ & 109.4 & $\mathrm{C} 27-\mathrm{C} 28-\mathrm{H} 28 \mathrm{~A}$ & 109.3 \\
\hline $\mathrm{C} 9-\mathrm{C} 8-\mathrm{H} 8 \mathrm{~B}$ & 109.4 & $\mathrm{C} 29-\mathrm{C} 28-\mathrm{H} 28 \mathrm{~B}$ & 109.3 \\
\hline $\mathrm{C} 7-\mathrm{C} 8-\mathrm{H} 8 \mathrm{~B}$ & 109.4 & $\mathrm{C} 27-\mathrm{C} 28-\mathrm{H} 28 \mathrm{~B}$ & 109.3 \\
\hline $\mathrm{H} 8 \mathrm{~A}-\mathrm{C} 8-\mathrm{H} 8 \mathrm{~B}$ & 108.0 & $\mathrm{H} 28 \mathrm{~A}-\mathrm{C} 28-\mathrm{H} 28 \mathrm{~B}$ & 108.0 \\
\hline $\mathrm{C} 14-\mathrm{C} 9-\mathrm{C} 10$ & $118.5(2)$ & $\mathrm{C} 34-\mathrm{C} 29-\mathrm{C} 30$ & $118.0(2)$ \\
\hline
\end{tabular}




$\begin{array}{llll}\mathrm{C} 14-\mathrm{C} 9-\mathrm{C} 8 & 123.4(2) & \mathrm{C} 34-\mathrm{C} 29-\mathrm{C} 28 & 123.3(2) \\ \mathrm{C} 10-\mathrm{C} 9-\mathrm{C} 8 & 118.1(2) & \mathrm{C} 30-\mathrm{C} 29-\mathrm{C} 28 & 118.7(2) \\ \mathrm{C} 11-\mathrm{C} 10-\mathrm{C} 9 & 121.2(2) & \mathrm{C} 31-\mathrm{C} 30-\mathrm{C} 29 & 121.4(2) \\ \mathrm{C} 11-\mathrm{C} 10-\mathrm{N} 1 & 121.3(2) & \mathrm{C} 31-\mathrm{C} 30-\mathrm{N} 3 & 121.1(2) \\ \mathrm{C} 9-\mathrm{C} 10-\mathrm{N} 1 & 117.6(2) & \mathrm{C} 29-\mathrm{C} 30-\mathrm{N} 3 & 117.5(2) \\ \mathrm{C} 10-\mathrm{C} 11-\mathrm{C} 12 & 119.6(2) & \mathrm{C} 32-\mathrm{C} 31-\mathrm{C} 30 & 119.6(2) \\ \mathrm{C} 10-\mathrm{C} 11-\mathrm{H} 11 & 120.2 & \mathrm{C} 32-\mathrm{C} 31-\mathrm{H} 31 & 120.2 \\ \mathrm{C} 12-\mathrm{C} 11-\mathrm{H} 11 & 120.2 & \mathrm{C} 30-\mathrm{C} 31-\mathrm{H} 31 & 120.2 \\ \mathrm{C} 13-\mathrm{C} 12-\mathrm{C} 11 & 120.1(2) & \mathrm{C} 31-\mathrm{C} 32-\mathrm{C} 33 & 119.9(2) \\ \mathrm{C} 13-\mathrm{C} 12-\mathrm{H} 12 & 119.9 & \mathrm{C} 31-\mathrm{C} 32-\mathrm{H} 32 & 120.1 \\ \mathrm{C} 11-\mathrm{C} 12-\mathrm{H} 12 & 119.9 & \mathrm{C} 33-\mathrm{C} 32-\mathrm{H} 32 & 120.1(2) \\ \mathrm{C} 12-\mathrm{C} 13-\mathrm{C} 14 & 120.5(2) & \mathrm{C} 34-\mathrm{C} 33-\mathrm{C} 32 & 119.9 \\ \mathrm{C} 12-\mathrm{C} 13-\mathrm{H} 13 & 119.8 & \mathrm{C} 34-\mathrm{C} 33-\mathrm{H} 33 & 119.9 \\ \mathrm{C} 14-\mathrm{C} 13-\mathrm{H} 13 & 119.8 & \mathrm{C} 32-\mathrm{C} 33-\mathrm{H} 33 & 11.0(2) \\ \mathrm{C} 9-\mathrm{C} 14-\mathrm{C} 13 & 120.1(2) & \mathrm{C} 33-\mathrm{C} 34-\mathrm{C} 29 & 119.5 \\ \mathrm{C} 9-\mathrm{C} 14-\mathrm{H} 14 & 119.9 & \mathrm{C} 33-\mathrm{C} 34-\mathrm{H} 34 & 122.7(2) \\ \mathrm{C} 13-\mathrm{C} 14-\mathrm{H} 14 & 119.9 & \mathrm{C} 29-\mathrm{C} 34-\mathrm{H} 34 & 121.01(19) \\ \mathrm{O} 1-\mathrm{C} 15-\mathrm{N} 2 & 122.9(2) & \mathrm{O} 2-\mathrm{C} 35-\mathrm{N} 4 & 116.2(2) \\ \mathrm{O} 1-\mathrm{C} 15-\mathrm{N} 1 & 120.5(2) & \mathrm{O} 2-\mathrm{C} 35-\mathrm{N} 3 & \\ \mathrm{~N} 2-\mathrm{C} 15-\mathrm{N} 1 & 116.6(2) & \mathrm{N} 4-\mathrm{C} 35-\mathrm{N} 3 & \\ \end{array}$

Hydrogen-bond geometry $\left(\AA,{ }^{\circ}\right)$

\begin{tabular}{lllll}
\hline$D-\mathrm{H} \cdots A$ & $D-\mathrm{H}$ & $\mathrm{H} \cdots A$ & $D \cdots A$ & $D-\mathrm{H} \cdots A$ \\
\hline $\mathrm{N} 2-\mathrm{H} 2 B \cdots \mathrm{O} 2^{\mathrm{i}}$ & $0.92(2)$ & $2.02(3)$ & $2.800(3)$ & $142.7(19)$ \\
$\mathrm{N} 4-\mathrm{H} 4 B \cdots \mathrm{O} 1$ & $0.86(2)$ & $2.11(3)$ & $2.801(3)$ & $137.4(19)$ \\
$\mathrm{N} 2-\mathrm{H} 2 A \cdots C g 4$ & $0.89(3)$ & $3.01(3)$ & $3.862(3)$ & $162(2)$ \\
$\mathrm{N} 4-\mathrm{H} 4 A \cdots C g 2^{\mathrm{ii}}$ & $0.90(3)$ & $2.89(3)$ & $3.765(3)$ & $166(2)$ \\
\hline
\end{tabular}

Symmetry codes: (i) $x, y-1, z$; (ii) $x, y+1, z$. 